\title{
Phenylene Bis-Diphenyltriazine (TriAsorB), a new sunfilter protecting the skin against both UVB + UVA and blue light radiations
}

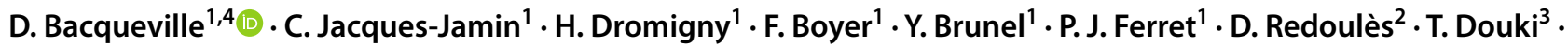 \\ S. Bessou-Touya ${ }^{1} \cdot$ H. Duplan ${ }^{1}$
}

Received: 3 June 2021 / Accepted: 5 October 2021 / Published online: 13 October 2021

(c) The Author(s), under exclusive licence to European Photochemistry Association, European Society for Photobiology 2021

\begin{abstract}
Sunlight induces actinic keratosis, skin cancers and photoaging. Photoprotection is thus a major issue in public health to prevent the harmful effects of solar ultraviolet (UV) radiations. Recent data have shown that the visible (VIS) and infrared (IR) radiations can lead to skin damage by oxidative stress, suggesting that a balanced protection across the entire spectrum of sunlight is necessary to prevent cutaneous alterations. In this context, we developed a new generation of sunfilter called

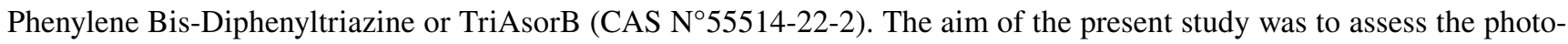
protective efficacy of TriAsorB from UV to IR light. Spectrophotometric assays were performed to measure absorption and reflectance of TriAsorB in the different spectral ranges of sunlight: UV, VIS including blue light or high energy visible (HEV) and IR. DNA damage was evaluated using reconstructed human epidermis (RHE): 8-hydroxy-2'-deoxyguanosine (8OHdG) in response to HEV exposure, pyrimidine dimers (CPDs) and (6-4) photoproducts following solar-simulated radiation (SSR). TriAsorB is a broad spectrum UVB + UVA filter including long UVA. Interestingly, it also absorbs VIS radiations, especially in the HEV region. These radiations are also reflected. Protection in the IR spectral range is weak. Furthermore, the sunfilter specifically protects the skin against the oxidative lesions $8 \mathrm{OHdG}$ induced by HEV and prevents SSR-induced DNA damage. Thus, TriAsorB is an innovative sunfilter that might be used in sun care products for skin photoprotection from UV to VIS radiations. Finally, it prevents sunlight genotoxicity and protected the skin against solar radiations, especially blue light.
\end{abstract}

Keywords Sunlight $\cdot$ Skin photoprotection $\cdot$ Sunscreen $\cdot$ Genoprotection $\cdot$ Oxidation

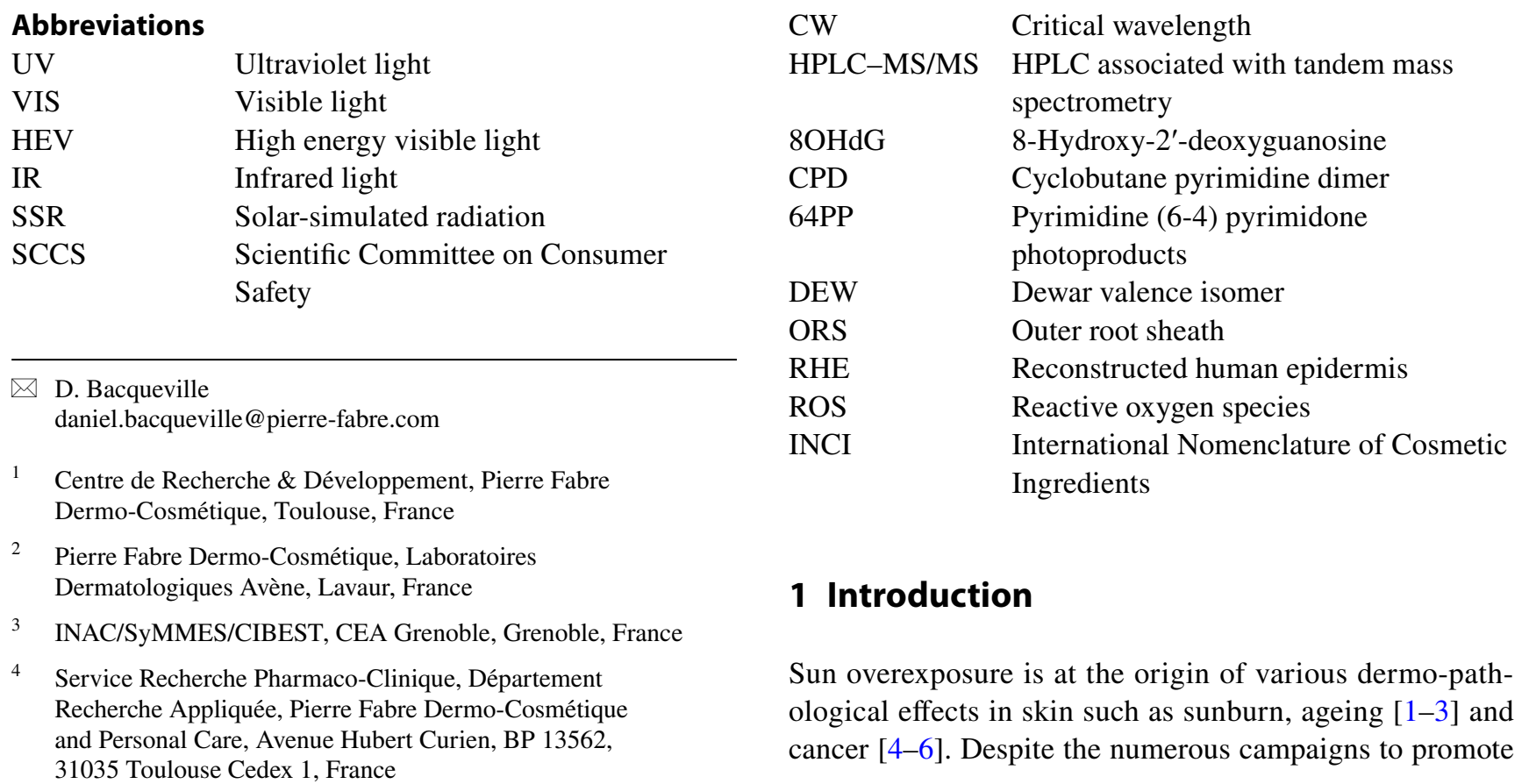


excessive sun avoidance, the exposure of the general public is still increasing in western countries for recreational and esthetic purposes [7]. Outdoor work is another large source of occupational exposure to solar radiation. With over 1,000,000 new cases and 65,000 deaths estimated globally, and incidence rates around two times higher among men than among women, nonmelanoma skin cancer is the most frequently diagnosed cancer in North America, Australia and New Zealand, the countries with the highest incidence rates worldwide in men and women, respectively. Western Europe incidence rates has been reported with 34.1 and 18.8 cases per year per 100,000 for men and women, respectively (GLOBOCAN 2018) [8-11]. Limiting the damage induced by solar radiation is thus a major issue in public health. In this context, sunscreens appear as an appropriate photoprotection strategy to reduces the radiation dose reaching both the dermis and epidermis, and thereby to limit the onset of the biological and biochemical processes leading to pathological conditions [12-14].

Research have been focused on the harmful effects of ultraviolet (UV) light that has the highest photon energy and the biggest impact on skin although this waveband accounts for only $5 \%$ of the solar spectrum. It is well known that UVB (290-320 nm) induces erythema, pigmentation, immunosuppression, alterations of extracellular matrix with collagen/ elastin network remodeling, but also initiate carcinogenesis and cause specific mutation fingerprint (cytidine to thymidine transition) as a result of photolesions in DNA [15]. The most frequent photoproducts are cyclobutane pyrimidine dimers (CPD), pyrimidine (6-4) pyrimidines (64PP) and their Dewar valence isomers (Dew) [16-19]. These result in structural damage to the DNA helix inhibiting DNA replication and transcription and generation of mutations. However, UVA (320-400 nm), especially the longest forms known as UVA1 (340-400 nm), are also able to induce the formation of DNA lesions even if they are a lot less powerful than UVB. Moreover, it is established that a part of the DNA damage induced by UVA is mediated by the generation of reactive oxygen species (ROS) in sun-exposed skin [20,21]. Thus, sunscreens have been initially developed to protect the skin against the UVB rays and their efficacy was extended to UVA to provide a broad spectrum UVB + A photoprotection for the consumers. Epidemiological studies have shown that regular sunscreen use could significantly reduce the risk of skin cancer [22]. A 4.5-year community-based randomized controlled trial conducted at Nambour, Australia, has been widely cited to show the efficacy of daily use of a broad-spectrum SPF15 $5^{+}$sunscreen in protecting against skin cancers in adult [23-25]. Although the efficacy of sun care products has greatly increased over the years with about 30 sunfilters authorized in Europe (both mineral and organic filters), it is now recognized that sunscreens must also provide a balanced protection against the entire solar spectrum [26,
27]. Indeed, recent data have highlighted that both the visible (VIS, 400-700 nm) and the infrared (IR, 700-2500 nm) wavebands could have deleterious effects for the skin. The VIS spectrum represents more than $50 \%$ of solar radiation and these wavelengths penetrate in the deepest parts of the dermis. Zastrow et al. [28, 29] have shown using electron spin resonance spectroscopy that $50 \%$ of the total skin oxidative burden was generated by VIS light but also near-IR radiation. The VIS and near-IR light could contribute to premature skin ageing or skin cancer by oxidative pathways. However, VIS and near-IR light can both generate bursts of reactive oxygen species that are beneficial rather than detrimental since they are broadly used in Dermatology to treat skin diseases (acne, psoriasis, scars...) or cancer by photodynamic therapy [30].

Although initially added into formulations to prevent UV-induced oxidative stress [20,21], antioxidants, such as vitamins $\mathrm{C}$ and $\mathrm{E}$ could therefore be also useful to fight the oxidative stress generated by the VIS light. Moreover, the blue light (400-450 $\mathrm{nm}$ ), also known as high-energy visible (HEV) light, has also recently received much attention [31], and it was shown to induce anti-proliferative [32] and oxidative stress-associated effects [33-35], disruption of epidermal barrier permeability [36], inflammation [37] and a pronounced and longer lasting hyperpigmentation [38]. More recently, blue light was also associated to a disruption of the circadian rhythm in skin cells [39]. It is of note that green and red wavelengths are considered less cytotoxic and damaging than blue light. In addition to the sun, there are multiple artificial sources of blue light, including electronic devices, fluorescent lamps or light-emitted diodes (LED). IR light also affects the skin and perturbates the extracellular matrix homoeostasis by degrading dermal connective tissue via oxidative induction of the metalloprotease MMP1 that leads to wrinkle formation and photoaging [40-42]. This oxidative pathway could take place at high IR intensity when using artificial light sources but not likely under sun exposure. Thus, the biological effects of VIS and IR radiations are now emerging although the UV radiations remain key players in photodermatology.

In this context, it is obvious that a large spectrum photoprotection beyond UV radiation remains a major challenge in sun care and that the development of innovative full spectrum sunfilters might help to fight against the harmful effects of the sun. Therefore, a new sunfilter from the 1,2,4-triazine family was recently described for improving performance and efficacy in solar protection. The members of the triazine family are heterocyclic compounds bearing triazine scaffold where three carbon-hydrogen units of benzene ring are replaced by nitrogen atoms [43-45]. The filter was 5,6,5',6'-tetraphenyl-3,3'-(1,4-phenylene)bis[1,2,4Triazine] and was called TriAsorB (CAS N ${ }^{\circ} 55514-22-2$ ). It was approved by the Scientific Committee on Consumer 
Safety (SCCS) and its toxicological dossier is available on the European Commission website (https://echa.europa.eu/ registration-dossier/-/registered-dossier/6106). Standard protocols were done according to guidelines and/or Good Laboratory Practices and it was concluded that TriAsorB is considered safe for use as a sunfilter in sunscreen products at a concentration up to 5\%. The structure of TriAsorB and an overview of the different assays performed to evaluate its safety are presented in Fig. 1. The aim of the present work was to assess the performance and efficacy of TriAsorB in solar protection. The studies were focused on the optical properties of the sunfilter from UV to IR light (290 to $2500 \mathrm{~nm}$ ), especially in the long UVA and the blue light/ HEV spectral ranges. Moreover, photoprotective efficacy of TriAsorB was compared to those of Tinosorb $S^{\circledR}$, a broadspectrum UVB + A sunfilter currently used in sun care products. DNA damage was also evaluated using reconstructed human epidermis (RHE). 8-hydroxy-2'-deoxyguanosine (8OHdG) was immunodetected in response to HEV exposure to assess TriAsorB photoprotection against the oxidative lesions induced by blue light. Finally, TriAsorB genoprotection was analyzed by measuring cyclobutane pyrimidine dimers (CPDs), pyrimidine (6-4) pyrimidone photoproducts (64PP) and their Dewar valence isomers (Dew) following solar-simulated radiation (SSR, UV + VIS radiations).

\section{Experimental}

\subsection{Physico-chemical characterization and cosmetic formulations}

TriAsorB empirical formula is $\mathrm{C}_{36} \mathrm{H}_{24} \mathrm{~N}_{6}$ and its molecular weight is $540.6 \mathrm{~g} \mathrm{~mol}^{-1}$. The structural formula is presented in Fig. 1 and it is of note that the sunfilter is a solid yellow powder which is highly pure and insoluble in a wide range of hydrophilic as well as lipophilic solvents (DMSO, PEG400). The $\log P_{\mathrm{o} / \mathrm{w}}$ is very high with a value of 10.5 , and the specific extinction coefficient $\varepsilon$ is equal to $52,492 \mathrm{~L} \mathrm{~mol}^{-1} \mathrm{~cm}^{-1}$ $(10 \mu \mathrm{g} / \mathrm{mL}$ in dichloromethane at $329 \mathrm{~nm})$.

The sunfilter was suspended at $40-50 \%$ in water and formulated as a cosmetic ingredient by wet grinding with an emulsifier (Eumulgin L, about $4.5 \%$ ) and a preservative (Benzoic acid, $0.2-0.3 \%$ ). Thus, the preparation is a $40-50 \%$ aqueous suspension of the finely dispersed active substance. It was stable for at least 18 months at room temperature and presented a high photostability ( $>98 \%$ ) according ICH Topic Q1B. Laser diffraction technology was used to determine the specific size of the particles and studies were performed using Mastersizer 2000 (Malvern Instruments Ltd) and Horiba LA950 (Horiba Scientific). Granulometry was also studied by electronic microscopy.
Fig. 1 TriAsorB structure, physico-chemical characterization and overview of toxicological assays to evaluate its safety

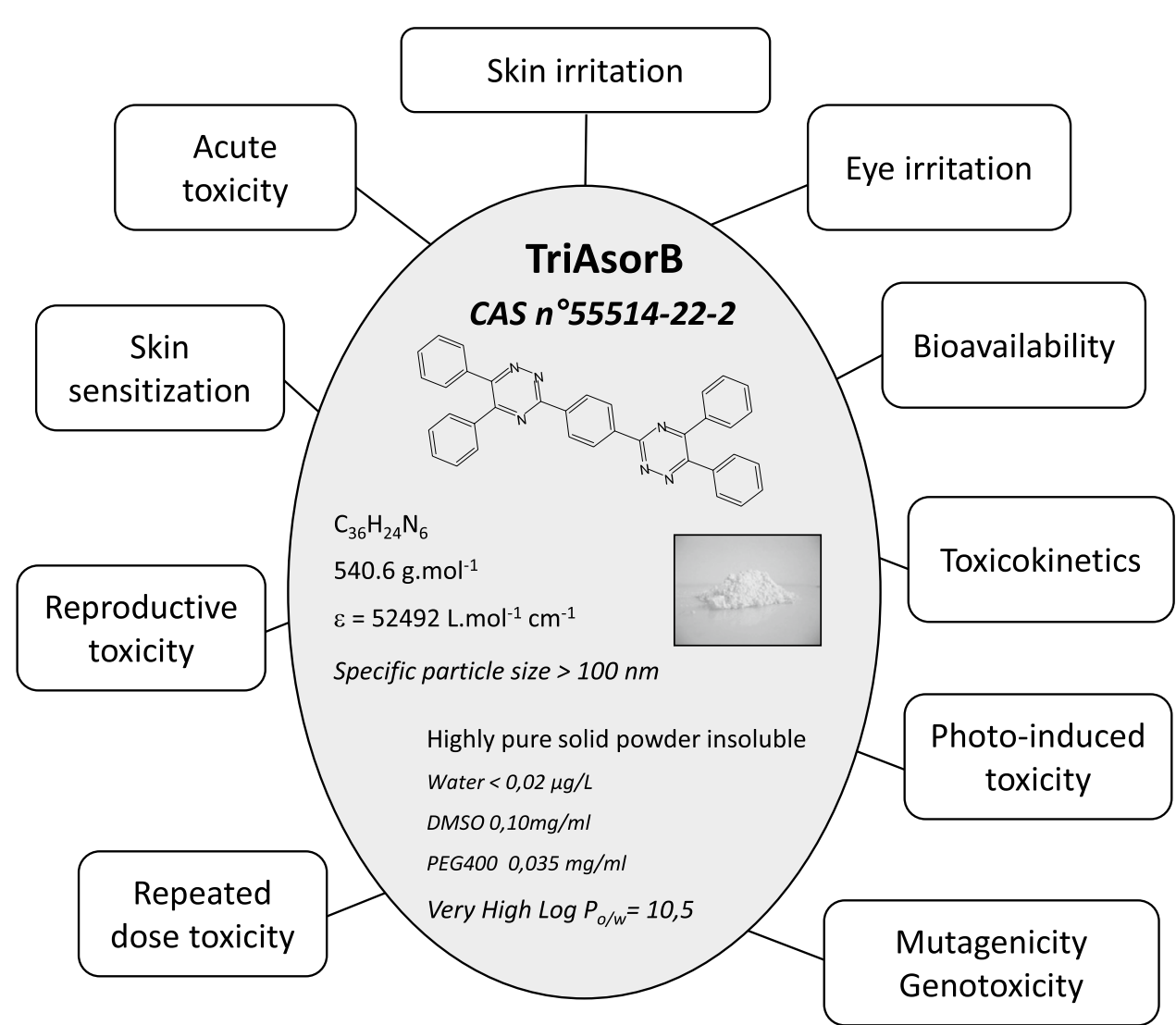


TriAsorB cosmetic ingredient was then formulated at $5 \%$ in a control formulation for the different experiments. Control formulation was prepared according the following INCI: Avene thermal spring water (Avene aqua), C12-15 alkyl benzoate, caprylic/capric triglyceride, dicaprylyl carbonate, glycerin, potassium cetyl phosphate, stearyl alcohol, VP/eicosane copolymer, benzoic acid, glyceryl behenate, glyceryl dibehenate, glyceryl stearate, PEG-100 stearate, phenoxyethanol, polyacrylate-13, polyisobutene, polysorbate 20 , sorbitan isostearate, tocopherol, tribehenin, water (aqua), xanthan gum. In addition, the broad-spectrum UV filter bisethylhexyloxyphenol methoxyphenyl triazine (Tinosorb $S^{\circledR}$ from BASF SE, Ludwigshafen Germany: CAS $N^{\circ} 187393-$ $\left.00-6, \mathrm{C}_{38} \mathrm{H}_{49} \mathrm{~N}_{3} \mathrm{O}_{5}, 627.8 \mathrm{~g} \mathrm{~mol}^{-1}, \log P_{\mathrm{o} / \mathrm{w}}=10.4\right)$ was also prepared at $5 \%$ in the same control formulation for comparison with TriAsorB. Finally, TriAsorB and Tinosorb $S^{\circledR}$ were mixed to test the efficacy of their association in the genoprotection studies.

\subsection{Spectrophotometric assays from UV to IR spectral range}

The photoprotective efficacy of TriAsorB from UV to IR was evaluated by spectrophotometric assays according initial report by Diffey and Farr [46]. Figure 2 presents the possible optical properties of a sunfilter following exposure to a light source: Absorption, Reflectance and Transmittance.

Absorption studies were performed using a spectrophotometer JASCO V-770 and automated formulation spreading on PMMA/Quartz plates according in vitro UVA-PF standard approach (ISO 24443) and COLIPA method [47]. The test is based on the assessment of UV transmittance through

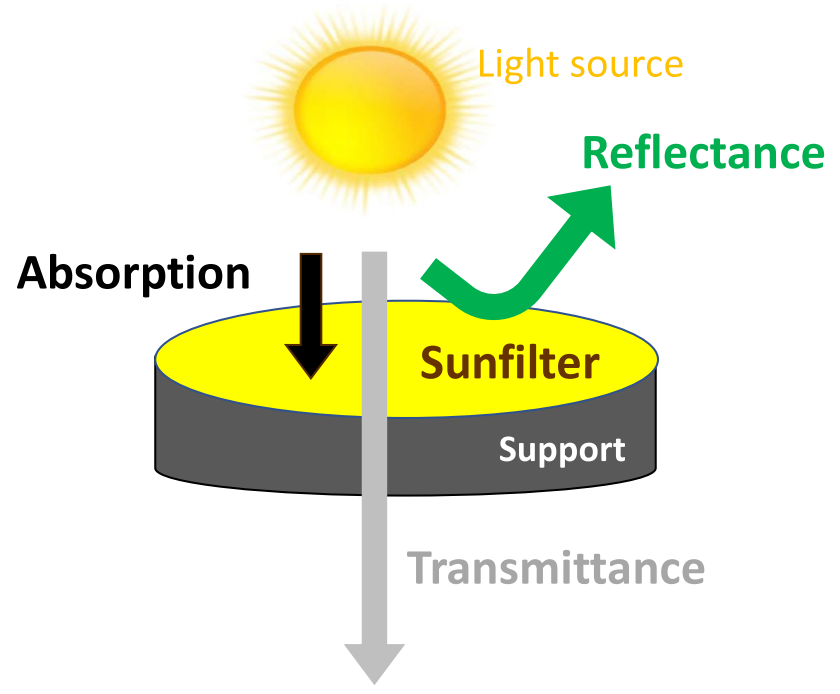

Fig. 2 Possible optical properties of sunfilter including absorption, reflectance and transmittance a thin film of the sun product sample, spread on the two substrates after exposure to a controlled dose of UV radiation, from a defined UV source. Then, mathematical adjustment of the absorbance spectrum (following UV exposure) to obtain a single absorbance curve from the UV to the IR. The $\lambda$ considered as the critical wavelength $(\mathrm{CW})$ is equal to $90 \%$ of the area under the absorption curve of the total area between 290 and $400 \mathrm{~nm}$ for $\lambda_{\mathrm{UV}-\mathrm{CW}}$, between 290 and $700 \mathrm{~nm}$ for $\lambda_{\text {Vis-CW }}$ and between 290 and 2500 for $\lambda_{\text {IR-CW}}$.

Reflectance was measured in the region 190-2500 nm (from UV to IR) using a UV/VIS/IR spectrometer Lambda 19 fitted with $60 \mathrm{~mm}$ integrating sphere operating in doublebeam mode (Perkin Elmer) and manual spreading on QX quartz slide. Reflectance measurements include total and diffuse reflectance at an incident angle of $8^{\circ}$. A $10^{-4} \mathrm{~g}$ precision laboratory balance was used to control deposited product weigh (Mettler). Transmittance values were transformed in Absorbance values by applying formula: $A=\log 10(1 / T)$.

\subsection{Oxidation protection evaluation in RHE exposed to blue light radiation}

Human epidermis was produced from primary normal human keratinocytes after 8 days of air exposure. Keratinocytes were isolated from abdominal dermolipectomy and obtained from 3 Caucasian healthy subjects with no skin disease history ( 31,35 and 51 years old women).

Oxidation protection study was done according to work published by Duteil and al. [48] using a blue light LED exposure (Kessil PR160). The wavelength was ranged at $427 \pm 30 \mathrm{~nm}$ including HEV light and the RHE received a dose at $80 \mathrm{~J} / \mathrm{cm}^{2}$. The LED power was about $176 \mathrm{~mW} /$ $\mathrm{cm}^{2}$ and the irradiation time was $75 \mathrm{~min}$ at a distance of $10 \mathrm{~cm}$. No variation of temperature was observed during the experiment.

Photoprotection assays were assessed by topical application of TriAsorB or control formulation at $2 \mathrm{mg} / \mathrm{cm}^{2}$ according to Cosmetics Europe recommendations. Sample were harvested $24 \mathrm{~h}$ after blue light exposure for immunohistochemical analysis from paraffin sections according a routine antigen retrieval method with citrate buffer [49]. Oxidation was evaluated using a monoclonal antibody directed against 8-hydroxy-2'-deoxyguanosine (MOG020P, clone N45.1, 1/20, Jaica) with overnight incubation at $4{ }^{\circ} \mathrm{C}$. Fluorescent detection was achieved with Alexa Fluor 555 goat anti-mouse IgG (A21424, 1/300, Life Technologies). Slides were mounted using Prolong Gold Antifade Reagent containing DAPI (Life Technologies) and the fluorescence signal was observed using a Nikon A1R + confocal microscope. Pictures were acquired and signal intensity was quantified using Nikon NIS elements. Results were expressed as the mean ratio 8-OHdG/ 
DAPI \pm SEM ( $n=10-15$ images $)$ and ANOVA test was performed for statistics with $* p<0.02$ and $* * * * p<0.0001$ (Graphpad Prism 9).

\subsection{Genoprotection evaluation in RHE exposed to solar-simulated radiation}

Human epidermis was reconstructed according to the protocol described by Guiraud et al. [50] from hair follicles obtained from 3 healthy Caucasian volunteers who had given their informed consent and with no skin disease history and no hair loss (21, 29 and 37 years old men). Briefly, outer root sheath (ORS) cells were isolated, plated on cell culture inserts and lifted to the air-medium interface for 16 days to differentiate the tissue into a functional multilayered epidermis [51].

Genoprotection study was done according to the previously described protocol [52]. Briefly, epidermis was irradiated by SSR using a Suntest CPS $^{+}$chamber (ATLAS Material Testing Technology BV, Moussy le Neuf, France) equipped with an NXE 1500 Xenon lamp, and fitted with a UV filter to eliminate wavelengths less than $290 \mathrm{~nm}$. Irradiance in UV spectra, measured by a recognized organization (Opto.cal gmbh), was about $70 \mathrm{~W} / \mathrm{m}^{2}$ from 290 to $400 \mathrm{~nm}$. Skin models were exposed to a single acute UV dose at $16.5 \mathrm{~J} / \mathrm{cm}^{2}$ (about $45 \mathrm{~min}$ ) and the irradiation chamber was maintained at $37^{\circ} \mathrm{C}$ using ice-cold water and airflow [49, 52]. Skin samples were harvested just after irradiation to quantify DNA lesions. Photoprotection assays were performed by topical application of TriAsorB formulations at $2 \mathrm{mg} / \mathrm{cm}^{2} 1 \mathrm{~h}$ before SSR irradiation according to Cosmetics Europe recommendations. Control formulation, Tinosorb $S^{\circledR}$ formulation and TriAsorB + Tinosorb $\mathrm{S}^{\circledR}$ formulation were applied in the same experimental conditions.

Pyrimidine dimers were quantified by HPLC-MS/MS spectrometry as previously described $[52,53]$. Briefly, the sample was crushed and after treatment by a mixture of RNase A and RNase T1, DNA was recovered by precipitation. It was then enzymatically hydrolyzed using endonucleases, exonucleases and phosphatases. The hydrolyzed mixture was then injected on the HPLC system. A UV detector was used to quantify unmodified nucleosides and thus the amount of analyzed DNA. Photoproducts were individually quantified using a triple quadrupolar mass spectrometer (MS) operated in the reaction monitoring mode. Cyclobutane pyrimidine dimers (CPD), pyrimidine (6-4) pyrimidone photoproducts (64PP) and their Dewar valence isomers were targeted. Results were expressed as mean \pm SEM number of photoproducts per million bases. Experiments were performed from 3 independent donors each in triplicate tissues and ANOVA test was performed for statistics with $p>0.05$ (ns, no significance) and $* * * p<0.001$.

\section{Results and discussion}

\subsection{Physico-chemical characterization and cosmetic formulations of TriAsorB}

TriAsorB belongs to the 1,2,4-triazine family [43-45]. This chemical group has six membered rings bearing three nitrogens as shown in Fig. 1. It has been widely described as scaffold of many biological active compounds, natural or synthetic, with a great variety of pharmacological effects, especially active as anti-tumor agents, anti-microbial and anti-inflammatory agents. In addition, the presence of multiple conjugated unsaturated heterocycles allows for electronic resonance properties and interactions with light and therefore define this triazine has a chromophore for sunlight.

Since TriAsorB is intended to be used as a broad-spectrum sunfilter in sunscreen products, its physio-chemical properties were studied by different techniques and the raw material was formulated as a cosmetic ingredient as indicated in the Experimental section. The data showed that TriAsorB is a yellow powder and has a high molecular weight $\left(>500 \mathrm{~g} \mathrm{~mol}^{-1}\right)$ that is theoretically predictive of a low penetration through the skin. In addition, it is insoluble in a wide range of hydrophilic as well as lipophilic solvents $\left(\log P_{\mathrm{o} / \mathrm{w}}=10.5\right)$, and formulation is clearly a fine dispersion of TriAsorB into an aqueous phase. Thus, TriAsorB can be classified as a non-soluble organic sunscreen. It is of note that laser diffraction technology evidenced that the particle size distribution obtained from the grinding process is beyond the threshold of $100 \mathrm{~nm}$. Therefore, the TriAsorB is a non-nanoparticle cosmetic ingredient. It has a high specific extinction coefficient $\varepsilon$ equals to $52,492 \mathrm{~L}$ $\mathrm{mol}^{-1} \mathrm{~cm}^{-1}(10 \mu \mathrm{g} / \mathrm{mL}$ in dichloromethane at $329 \mathrm{~nm})$. Finally, photostability assay showed that the filter is not light sensitive (greater than 98\%).

The safety of TriAsorB was previously assessed by SCCS as mentioned earlier and shown in Fig. 1. As example, sunfilter safety was evaluated on genotoxicity/phototoxicity assays but also acute toxicity and skin irritation, completed by availability analysis. Based on the data provided, calculation of margin of safety (MOS) was 980 , a value largely superior to the recognized safety limit of $M O S \geq 100$, and it was concluded that TriAsorB is considered safe for use as a sunfilter in sunscreen products at a concentration up to $5 \%$. In this respect, it was formulated as a cosmetic ingredient at $5 \%$ into a sun care formulation. A control formulation was also prepared for the different experiments.

\subsection{TriAsorB, a broad spectrum UVB + A and blue light sunfilter}

The photoprotective efficacy of TriAsorB was evaluated from UV to IR by spectrophotometric assays and 
was compared to those of Tinosorb $S^{\circledR}$, a broad spectrum $\mathrm{UVB}+$ A sunfilter. As shown in Fig. 2, it was important to determine whether TriAsorB has specific optical properties related to light absorption and/or reflection to provide skin protection against sun radiations. The solar spectrum was divided into three distinct spectral ranges: UV from 290 to $400 \mathrm{~nm}$, VIS from 400 to $700 \mathrm{~nm}$, and finally IR from 700 to $2500 \mathrm{~nm}$ [26-29]. The data from absorption studies are presented in Fig. 3 and showed that TriAsorB absorbs UVB (290-320 nm) but also UVA especially in the long UVA range also called UVA1 (340-400 nm). Control formulation presented no absorption along UV wavelengths. Thus, TriAsorB is a broad spectrum UVB + UVA filter. The critical wavelength for UV spectral range has a high value and was $\lambda_{\mathrm{UV}-\mathrm{CW}}=388 \mathrm{~nm}$, suggesting a balanced UVB + UVA protection. In vitro critical wavelength test is defined as the wavelength below which $90 \%$ of the total area under the UV absorbance curve. A critical wavelength of $370 \mathrm{~nm}$ or greater is required for sunscreens to be labeled as "broad spectrum" and the European Union recommends both a $\lambda_{\mathrm{UV}-\mathrm{CW}}>370 \mathrm{~nm}$ and UVA protection factor at least one third of the labeled sun protection factor (SPF) as the criterion for labeling as either UVA or broad-spectrum UVB + A protection [12-14]. As expected, Tinosorb $S^{\circledR}$ was able to absorb both UVB and UVA radiations with high efficacy (Fig. 3). However, its absorption sharply dropped from about $380 \mathrm{~nm}$ and it was not as powerful as TriAsorB to absorb long UVA. Its critical wavelength for UV spectral range was $\lambda_{\text {UV-CW }}=374 \mathrm{~nm}$. Thus, TriAsorB provides a better protection against long UVA than Tinosorb $S^{\circledR}$. This result is interesting since a few sunfilters in the dermo-cosmetic market have the capability to absorb both UVB and UVA radiations. In addition to Tinosorb $S^{\circledR}$, broad-spectrum $\mathrm{UVB}+$ A sunfilters approved in Europe are for example the
Tinosorb ${ }^{\circledR}$ M from BASF SE (Ludwigshafen Germany), Parsol ${ }^{\circledR}$ 1789/5000, trademark of DSM (Kaiseraugst, Switzerland), and Mexoryl ${ }^{\circledR}$ XL/SX from L'Oréal (Paris France). Moreover, the analysis of TriAsorB reflectance in the UV spectral range revealed negative values (Fig. 4), indicating that the filter is unable to reflect UV light but instead absorbs these radiations. Similar result was obtained for Tinosorb $S^{\circledR}$ as shown in Fig. 4.

Next, we decided to check the ability of TriAsorB to absorb the VIS light since its UV absorption presented a value of absorbance at about 0.3 unit at $400 \mathrm{~nm}$. The results are presented in Fig. 3. It appeared that the filter effectively absorbs VIS light especially in the region from 400 to $450 \mathrm{~nm}$ bandwidth. The absorbance decreased with an increasing wavelength and dropped at about 0.1 at $450 \mathrm{~nm}$ with a $\lambda_{\text {Vis-CW }}=594 \mathrm{~nm}$. The value of control formulation was $437 \mathrm{~nm}$. In addition, the analysis of reflectance in the VIS spectral range revealed that TriAsorB is also able to reflect HEV light with a peak of $8 \%$ at about $450 \mathrm{~nm}$ and a progressive decrease up to $700 \mathrm{~nm}$ (Fig. 4). In contrast, Tinosorb $S^{\circledR}$ was not able to absorb VIS light (Fig. 3) and its $\lambda_{\text {Vis-CW }}$ was calculated at $374 \mathrm{~nm}$. This filter was also unable to reflect HEV light as shown in Fig. 4 and therefore do not protect against VIS light.

Thus, these results clearly showed that TriAsorB presents the ability to absorb and reflect VIS light, especially in the 400 to $450 \mathrm{~nm}$ spectral range corresponding to the blue light. Interestingly, blue light has been recently called high energy visible light (HEV) and is associated with skin damage including pigmentation, inflammation, weakening of the epidermal barrier and aging effects [31-39]. Although the per-photon efficacy of blue light to oxidize glutathione was only $25 \%$ of UVA, blue light is more than twice as abundant in sunlight as UVA, suggesting the contribution of blue light
Fig. 3 Absorption of TriAsorB formulation in the UV and VIS spectral range. Data are expressed as Absorbance according to the wavelength in the specific spectral ranges: 290-400 nm for UV (UVB 290-320 nm, short UVA2 $320-340 \mathrm{~nm}$ and long UVA1 $340-400 \mathrm{~nm}$ ) and $400-700 \mathrm{~nm}$ for VIS spectrum with HEV/ blue light spectral range from 400 to $450 \mathrm{~nm}$. TriAsorB absorption was compared to those of the control formulation and Tinosorb $S^{\circledR}$. Both sunfilters were formulated at a $5 \%$ concentration

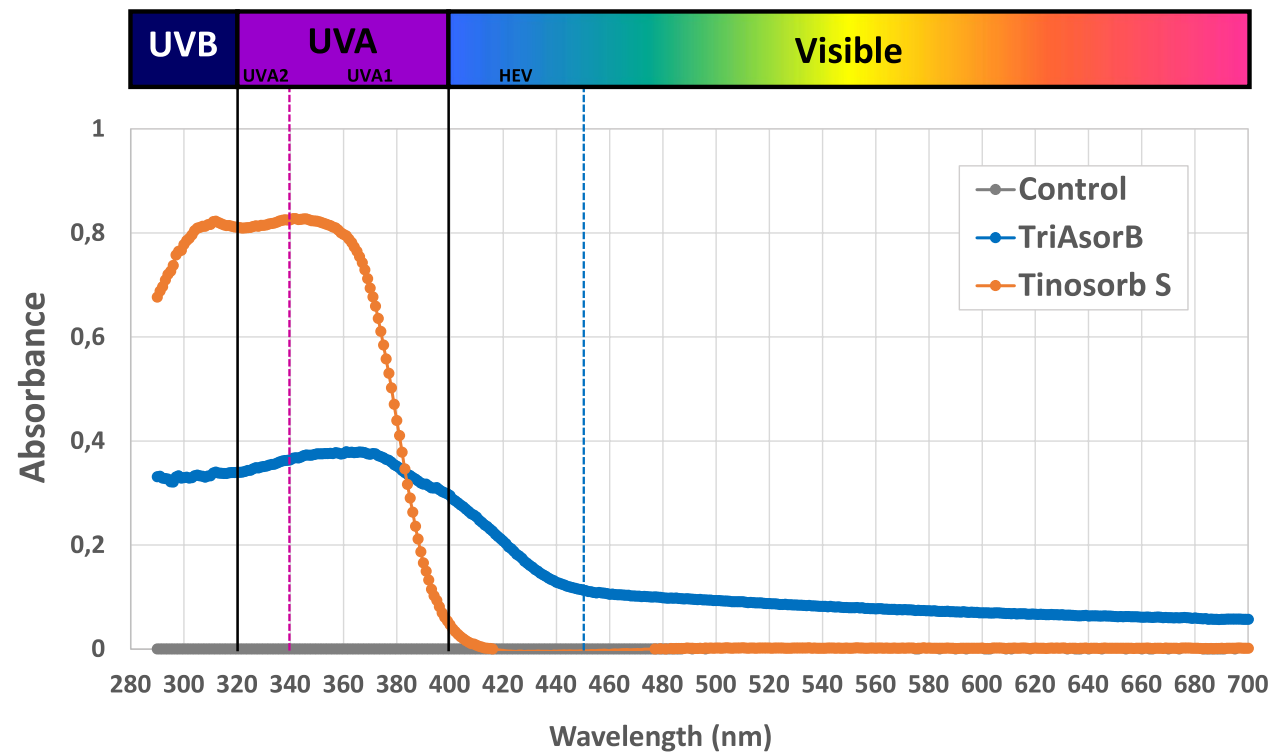

Wavelength $(\mathrm{nm})$ 
Fig. 4 Reflectance of TriAsorB formulation in the UV and VIS spectral range. Data are expressed as \% Reflectance according to the wavelength in the specific spectral ranges: 290-400 nm for UV (UVB 290-320 nm, short UVA2 $320-340 \mathrm{~nm}$ and long UVA1 $340-400 \mathrm{~nm}$ ) and 400-700 nm for VIS spectrum with HEV/ blue light spectral range from 400 to $450 \mathrm{~nm}$. TriAsorB absorption was compared to those of the control formulation and Tinosorb $S^{\circledR}$. Both sunfilters were formulated at a $5 \%$ concentration

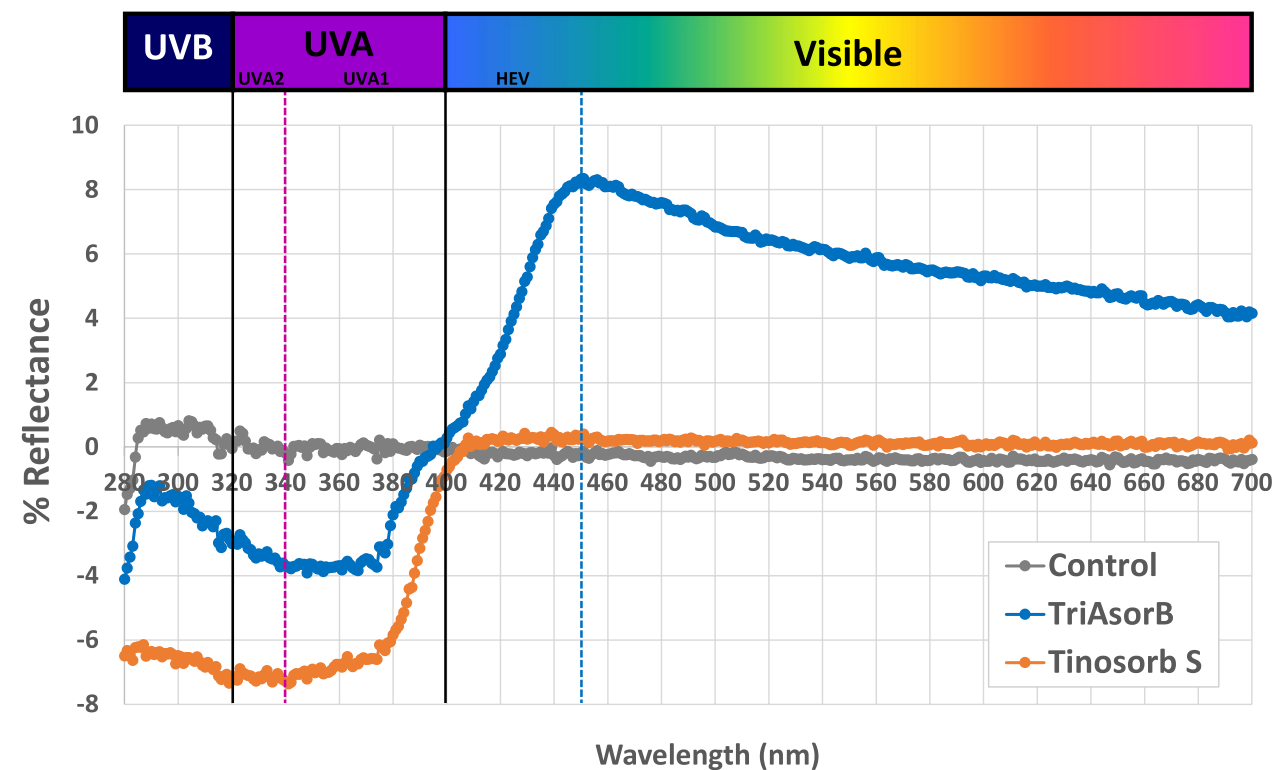

to ROS production might be about half that of UVA [33]. In addition to natural blue light source from the sun rays, consumers are exposed to artificial blue light. Indeed, they use more and more digital and electronic screens (television, cellular phones, laptops...) at work and at home. These devices use LED technology to improve brightness and clarity but this kind of technology emits strong blue light radiations. However, it is of note that artificial blue light from devices is at least two to three orders of magnitude less than natural blue light and that their biological effects on the skin are still poorly understood in real life conditions. Thus, blue light protection could be helpful to prevent tissue damage notably following excess sun exposure. In this specific case, the photoprotection of TriAsorB that covers the HEV spectral range may help to fight against the deleterious effects of these radiations.

In addition, sunscreen based on TriAsorB absorption/ reflection properties could also be effective to improve the treatment of photodermatoses with action spectrum in the VIS light range as for example solar urticaria [54], a mast cell-mediated disease, and erythropoietic protoporphyria that is associated with acute phototoxicity [55]. Moreover, it has been recently shown that sunscreen covering UV + VIS spectral range (and containing pigments) decreases severity index scores, colorimetric values and melanin assessments in melasma in comparison to UV only, suggesting that VIS light may play a role in the pathogenesis of this disease that is an acquired hyperpigmentation on sun-exposed areas, especially in women of darker skin types [56, 57]. It is of note that VIS/blue light also induces an oxidative stress that mediates the release of proinflammatory cytokines and matrix metalloproteinases aggravating skin photoaging. The addition of antioxidants such as vitamin $\mathrm{C}$ or $\mathrm{E}$ into sunscreen formulation could therefore downregulate the free radical generation induced by sunlight [20,21], and thus reinforces TriAsorB sunfilter efficacy to fight against tissue oxidation.

Finally, the optical properties of TriAsorB were studied in the IR spectral range from 700 to $2500 \mathrm{~nm}$. The data showed that the filter slightly protects from IR radiations with a low absorption with a $\lambda_{\text {IR-CW }}=1822 \mathrm{~nm}$ (Fig. 5 upper panel), but also a low reflectance with a maximum at 3\% (Fig. 5 lower panel). Thus, TriAsorB protection was weak against IR from the sunlight and it is doubtful that its low absorbance and reflectance in this spectral range has any beneficial effects, especially to decrease the IR-induced oxidative stress at the skin surface. Further experiments will be needed to prove this. Tinosorb $S^{\circledR}$ was also unable to block IR light (Fig. 5). However, it is important to note that the need for IR protection by sun care products is highly questionable [58] and the heat of these radiations provides an alert signal to avoid sunburn for consumers.

\subsection{TriAsorB, an antioxidant sunfilter against blue light radiation}

To provide further evidence that TriAsorB provides a biologically relevant skin photoprotection against blue light/ HEV spectrum radiations, we used a reconstructed human epidermal model. The spectral range was centered to $427 \mathrm{~nm}$ in the $\mathrm{HEV}$ region $(400-450 \mathrm{~nm})$ and the epidermis received a dose at $80 \mathrm{~J} / \mathrm{cm}^{2}$, corresponding to about $2 \mathrm{~h}$ and $30 \mathrm{~min}$ of sun exposure during summer [48]. After topical application of TriAsorB, 8-hydroxy-2'-deoxyguanosine (8OHdG) was detected by immunohistochemistry as a biomarker of oxidative DNA damage. Indeed, it is a product of oxidatively 
Fig. 5 Absorption and Reflectance of TriAsorB formulation in the IR spectral range. Data are expressed as Absorbance (upper panel) and \% Reflectance (lower panel) according to the wavelength in the specific IR spectral range from 700 to $2500 \mathrm{~nm}$. TriAsorB absorption and reflectance was compared to those of the control formulation and Tinosorb $S^{\circledR}$. Both sunfilters were formulated at a $5 \%$ concentration
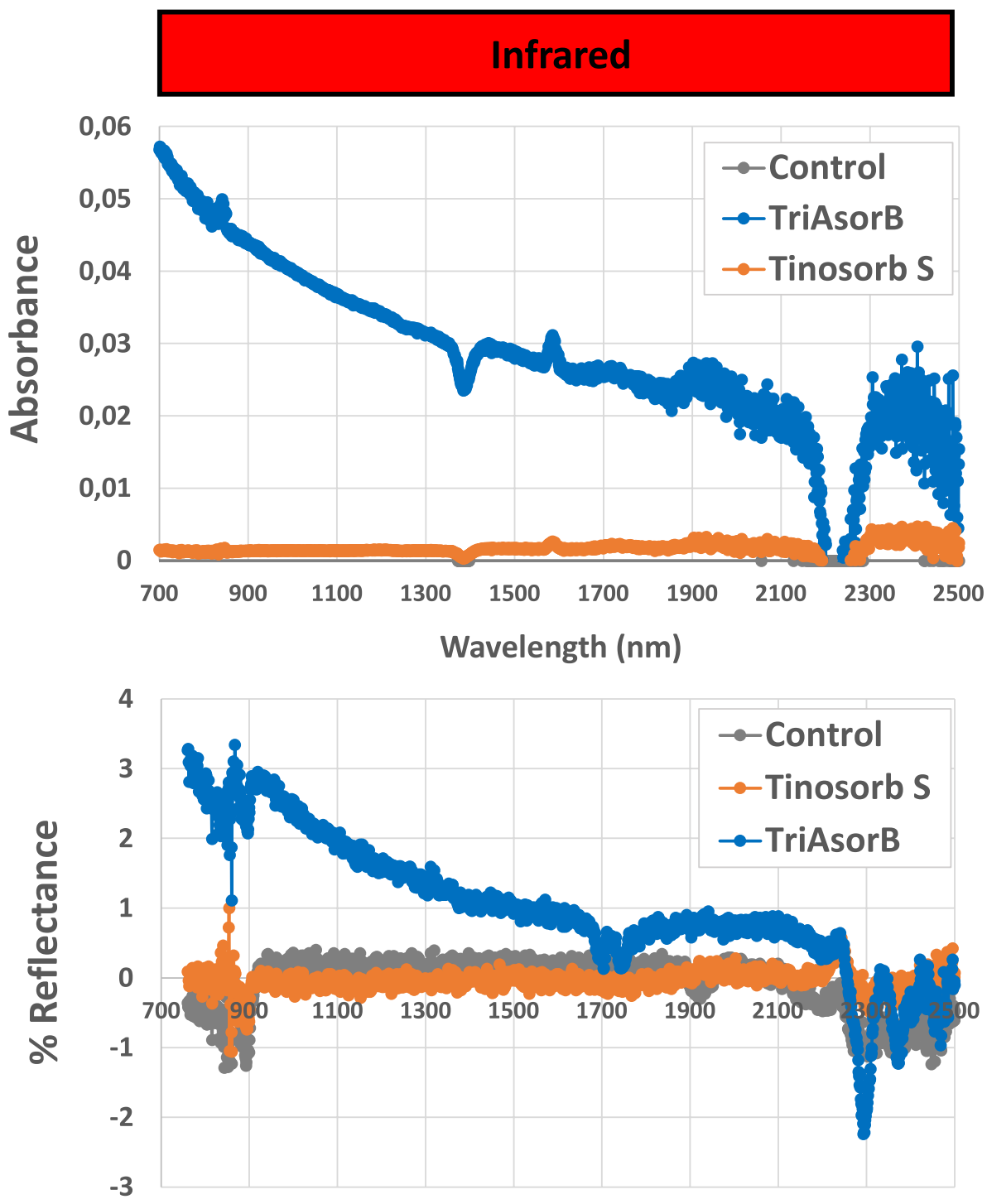

Wavelength (nm damaged DNA formed by hydroxy radical, singlet oxygen and direct photodynamic action. Representative pictures and signal quantification are presented in Fig. 6. Data showed that $8 \mathrm{OHdG}$ is weakly present in the skin not exposed to blue light (ratio $8 \mathrm{OHdG} / \mathrm{DAPI}=1.313 \pm 0.049$ ). In contrast, HEV radiation induced an increase of the fluorescent signal and therefore generated an oxidative stress in the RHE (ratio $8 \mathrm{OHdG} / \mathrm{DAPI}=2.531 \pm 0.162$ ). The data are in accordance with the recent findings by Chamayou-Robert et al. [59] who showed using COMET and micronucleus assays that a single blue light exposure at $415 \mathrm{~nm}$ generates both oxidized DNA bases and CPD that may turn into chromosome aberrations in human keratinocyte culture. A drop was observed when control formulation was applied before blue light exposure (ratio $8 \mathrm{OHdG} / \mathrm{DAPI}=1.653 \pm 0.074$ ). More interestingly, TriAsorB topical application allowed to maintain a low level of $8 \mathrm{OHdG}$ in the blue light-exposed epidermis that was similar with the non-irradiated condition (ratio 8OHdG/ DAPI $=1.111 \pm 0.090)$. The difference between control and TriAsorB formulations was significant $(p<0.02)$ and therefore the sunfilter provides a protection against blue light. In addition, it could be helpful to perform complementary biochemical assays to reinforce the sunfilter protection against the oxidative stress induced by blue light exposure. In this respect, it could be interesting to measure the activity of antioxidant enzymes such as superoxide dismutase or catalase but also to quantify the cellular glutathione (GSH) which is depleted and converted into GSSG after the quenching of reactive oxygen species [20,21].

Thus, TriAsorB provides an effective photoprotection against blue light-induced oxidation and as a result could be useful to fight against skin damage associated with blue 

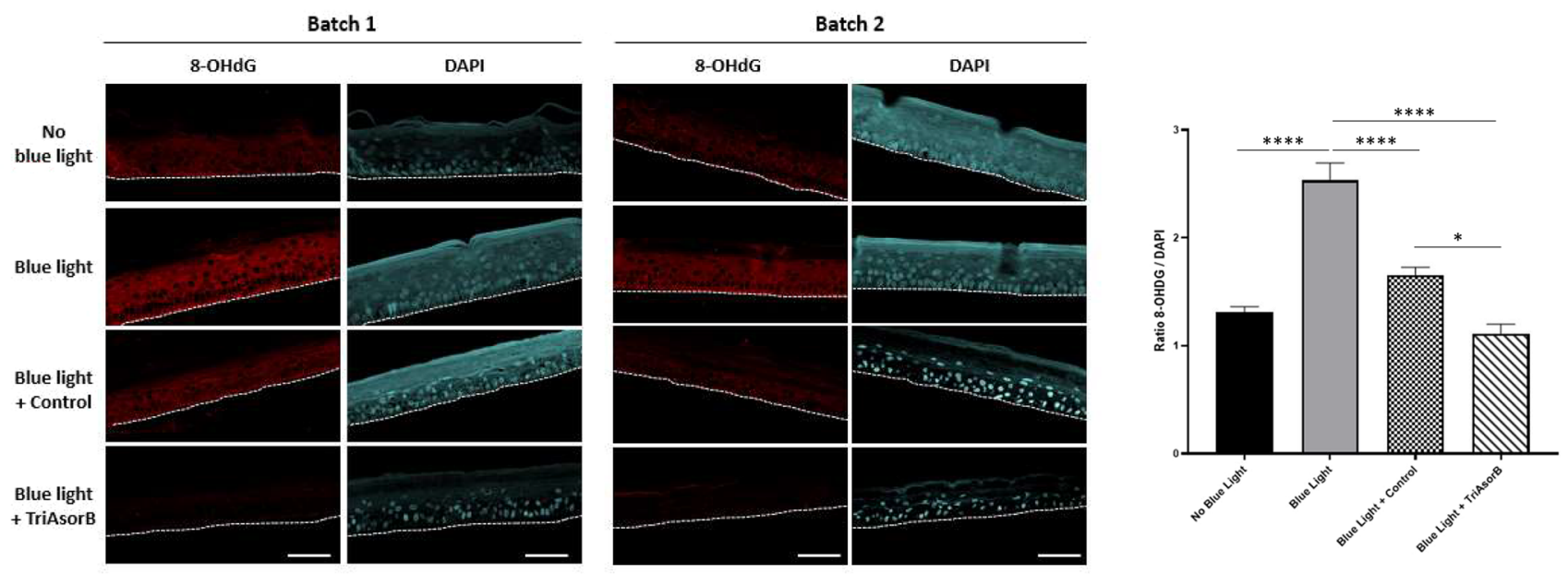

Fig. 6 Oxidation protection of TriAsorB after Blue light exposure in reconstructed human epidermis. Immunohistochemical staining of 8-hydroxy-2'-deoxyguanosine, $(8 \mathrm{OHdG})$ as oxidative stress marker. Signal was visualized using a red fluorescent detection and nuclei were stained in blue with DAPI. Representative pictures obtained

light including pigmentation, inflammation, epidermal barrier defect and photoaging [31-39].

\subsection{TriAsorB, a genoprotective sunfilter against solar-simulated radiation}

The above results suggest that TriAsorB is a broad-spectrum UVB + A and blue light photoprotector. Therefore, the next step was to study the performance of the sunfilter to protect the skin against UV + VIS light. Reconstructed human epidermis was exposed to an acute dose of solarsimulated radiation (SSR) that mimics UV + VIS spectrum of sunlight [52]. Genoprotection assays were performed after topical application of TriAsorB formulation. The data are presented in Fig. 7. SSR exposure induced the formation of DNA photoproducts with a high amount of TT CPDs accounting for about the half of total CPDs $(313.1 \pm 78.5$ lesions versus $531.7 \pm 130.1$ ). There was also generation of 64PP and Dewar isomers with nearly 200 lesions per million of bases. The DNA lesions are interesting markers because they are at the origin of skin cancer and they also directly reflect the amount of SSR photons that reach cutaneous cells after getting through the skin layers. CPD lesions have been associated to cytokine-mediated inflammation leading to erythema and concomitant immunosuppression and transition mutations, such as $\mathrm{C}$ (cytosine)/ $\mathrm{T}$ (thymine) or even $\mathrm{CC}$ / TT, which can lead to keratinocyte cancers [15-19].

The control formulation did not protect the skin against SSR-induced DNA lesions and the DNA lesion yield was identical to the SSR exposure (482.2 \pm 57.8 lesions, $9.32 \%$ inhibition not significant with $p>0.05$ ). Interestingly, TriAsorB prevented the formation of photoproducts with a total from two donors are shown. Dashed lines correspond to the membrane insert in RHE model. Scale bar $=50 \mu \mathrm{m}$. Signal quantification was expressed as the mean ratio $8-\mathrm{OHdG} / \mathrm{DAPI} \pm$ SEM. Statistical analysis is $* p<0.02$ and $* * * * p<0.0001$

CPD level at $260.1 \pm 77.1$ photoproducts/million bases and inhibited $51.08 \%$ of the DNA damage. All types of DNA lesions were protected from SSR radiations and data were significantly different $(p<0.001)$ for cyclobutane pyrimidine dimers (CPD), pyrimidine (6-4) pyrimidone photoproducts (64PP) and their Dewar valence isomers (Dew). It is of note that Kielbassa et al. [60, 61] showed in AS52 cells that most of these DNA lesions are due to direct excitation of DNA by the UV part of the SSR (290-500 nm) and that the yield of pyrimidine dimers decreases exponentially beyond $315 \mathrm{~nm}$ but are detectable even after irradiation at wavelengths $>360 \mathrm{~nm}$ in the UVA spectrum. Interestingly, they also demonstrated that oxidative DNA base modifications are generated at wavelengths up to about $340 \mathrm{~nm}$ in parallel with CPDs and at longer wavelengths with a maximum between 400 and $450 \mathrm{~nm}$. Thus, there are a wavelength dependence of oxidative DNA lesion induction and an increase of oxidative DNA damage in the HEV/blue light spectral range. Our data in Fig. 6 highlight this finding and the mass spectrometry approach used in our experiments gives evidence for TriAsorB efficacy in the UV spectral range rather than the VIS light. Finally, TriAsorB provides an effective genoprotection against SSR.

Sunfilters are used in combination to give a maximal photoprotection in sun care products. Therefore, last experiments aimed to show that the association TriAsorB + Tinosorb $\mathrm{S} \circledast$ could provide a very efficient genoprotection (Fig. 7). The data clearly showed that the sunfilters association strongly protects the skin with only $51.3 \pm 14.5$ lesions and an inhibition at $90.3 \%(p<0.001)$. Thus, the association of these two filters could be enough in preventing DNA lesions due to SSR irradiation in sun care product development. 
Fig. 7 Genoprotection of TriAsorB after SSR exposure in reconstructed human epidermis. Data are shown for all cyclobutane pyrimidine dimers (All CPD), TT CPD but also pyrimidine (6-4) pyrimidone photoproducts (64PP) and their Dewar valence isomers (Dew). DNA lesions were expressed as mean photoproducts per million bases \pm SEM. Statistical analysis is no significance (ns) with $p>0.05$ and $* * * p<0.001$

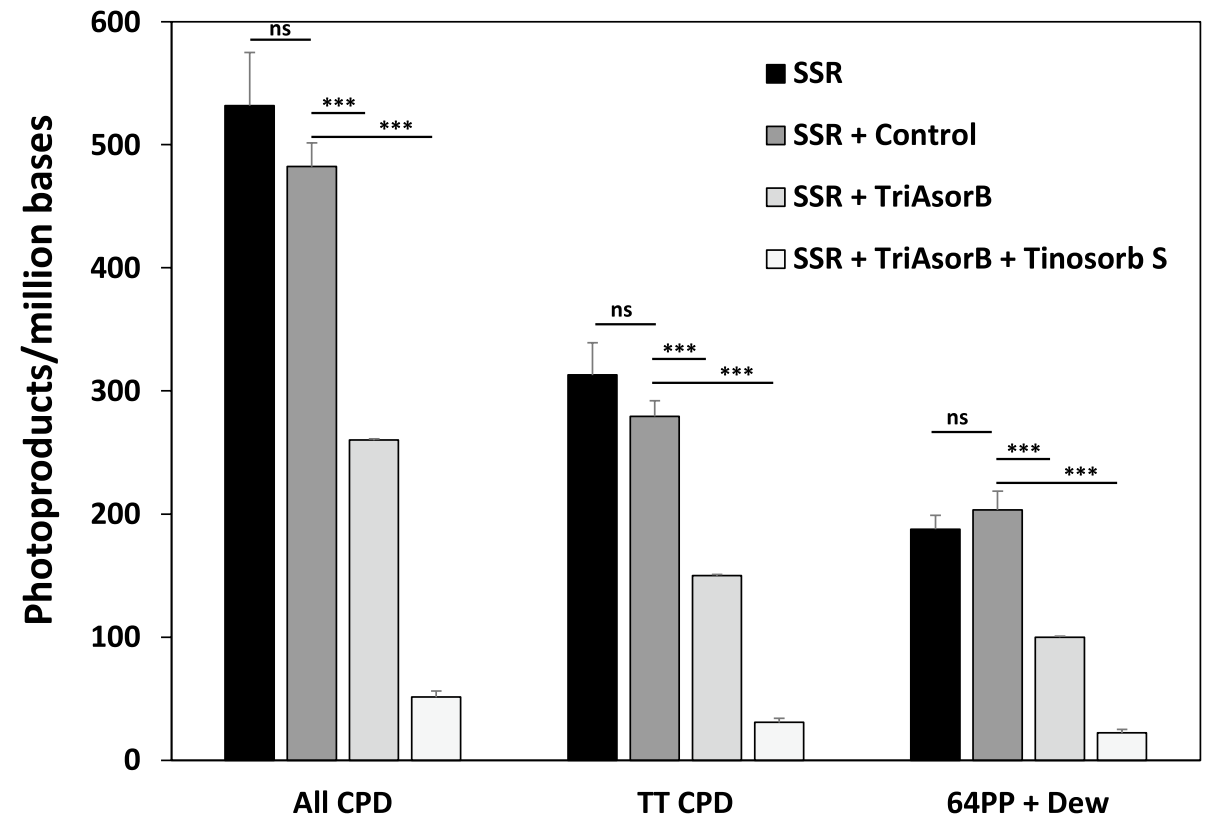

\section{Conclusions}

To conclude, TriAsorB has no reported risk to human health and represents a new generation of non-soluble organic sunscreen effective against UVB + UVA and blue light. It might be used in combination with other specific sunfilters, such Tinosorb $\mathrm{S}^{\circledR}$, but also with antioxidants (vitamin $\mathrm{C} / \mathrm{E}$ ) to provide a very efficient skin photoprotection from UV to blue light. TriAsorB prevents SSR-induced DNA damage and specifically protects the skin against the oxidative lesions induced by $\mathrm{HEV}$. It could be formulated in sun care products for maximal genoprotection to limit excessive sun damage. With sun exposure and non-melanoma skin cancers rising worldwide, TriAsorB alone or combined to existing sunfilters, may provide additional protection against longer wavelengths in the UVA/ blue light spectra. In this respect, clinical trials will be helpful to show the efficacy of TriAsorB-based sunscreen on a daily basis to significantly decrease the risk of skin cancers in adult.

Acknowledgements We are grateful to the following colleagues for their help in experiments: L. Duprat, A. Tourette, Frédéric Crépel, V. Périer and L. Cattuzzato from Pierre Fabre Dermo-Cosmétique. We also thank V. Georgescu, A. Couttet, S. Saint André and C. JeanDecoster from Avène laboratories, but also Alain Moga and Nicolas Almaric from Synelvia for performing blue light evaluation.

Funding The reported studies were funded by Pierre Fabre DermoCosmétique, France and Laboratoires Dermatologiques Avène.

\section{Declarations}

Conflict of interest All authors except T. Douki are employees of Pierre Fabre group, France. The authors report no other conflicts of interest associated with this work.

\section{References}

1. Yaar, M., \& Gilchrest, B. A. (2007). Photoageing: Mechanism, prevention and therapy. British Journal of Dermatology, 157, 874-887.

2. Naylor, E. C., Watson, R. E. B., \& Sherratt, M. J. (2011). Molecular aspects of skin ageing. Maturitas, 69, 249-256.

3. Lopez-Otin, C., Blasco, M. A., Partridge, L., Serrano, M., \& Kroemer, G. (2013). The hallmarks of aging. Cell, 153, 1194-1217.

4. Narayanan, D. L., Saladi, R. N., \& Fox, J. L. (2010). Ultraviolet radiation and skin cancer. International Journal of Dermatology, 49, 978-986.

5. Pfeifer, G. P., \& Besaratinia, A. (2012). UV wavelength dependent DNA damage and human non-melanoma and melanoma skin cancer. Photochemical \& Photobiological Sciences, 11, 90-97.

6. Ratushny, V., Gober, M. D., Hick, R., Ridky, T. W., \& Seykora, J. T. (2012). From keratinocyte to cancer: The pathogenesis and modeling of cutaneous squamous cell carcinoma. The Journal of Clinical Investigation, 122, 464-472.

7. Goulart, J. M., \& Wang, S. Q. (2010). Knowledge, motivation, and behavior patterns of the general public towards sun protection. Photochemical \& Photobiological Sciences, 9, 432-438.

8. Leiter, U., Eigentler, T., \& Garbe, C. (2014). Epidemiology of skin cancer. Advances in Experimental Medicine and Biology, $810,120-140$.

9. Apalla, Z., Lallas, A., Sotiriou, E., Lazaridou, E., \& Ioannides, D. (2017). Epidemiological trends in skin cancer. Dermatology Practical \& Conceptual, 7(2), 1-6.

10. Lai, V., Cranwell, W., \& Sinclair, R. (2018). Epidemiology of skin cancer in the mature patient. Clinics in Dermatology, 36, 167-176.

11. Bray, F., Ferlay, J., Soerjomataram, I., Siegel, R. L., Torre, L. A., \& Jemal, A. (2018). Global cancer statistics 2018: GLOBOCAN estimates of incidence and mortality worldwide for 36 cancers in 185 Countries. CA: A Cancer Journal of Clinicians, 68, 394-424.

12. Osterwalder, U., Sohn, M., \& Herzog, B. (2014). Global state of sunscreens. Photodermatology, Photoimmunology and Photomedicine, 30, 62-80. 
13. Iannacone, M. R., Hughes, M. C. B., \& Green, A. (2014). Effects of sunscreen on skin cancer and photoaging. Photodermatology, Photoimmunology and Photomedicine, 30, 55-61.

14. Young, A. R., Claveau, J., \& Rossi, A. B. (2017). Ultraviolet radiation and the skin: Photobiology and sunscreen photoprotection. Journal of the American Academy of Dermatology, 76, S100-109.

15. Ziegler, A., Leffel, D. J., Kunala, S., Sharma, H. W., Shapiro, P. E., Bale, A. E., \& Brash, D. E. (1993). Mutation hotspots due to sunlight in the p53 gene of nonmelanoma skin cancers. Proceedings of the National academy of Sciences of the United States of America, 90, 4216-4220.

16. Cadet, J., \& Douki, T. (2018). Formation of UV-induced DNA damage contributing to skin cancer development. Photochemical \& Photobiological Sciences, 17, 1816-1841.

17. Perdiz, D., Grof, P., Mezzina, M., Nikaido, O., Moustacchi, E., \& Sage, E. (2000). Distribution and repair of bipyrimidine photoproducts in solar UV-irradiated mammalian cells. Possible role of Dewar photoproducts in solar mutagenesis. Journal of Biological Chemistry, 275, 26732-26742.

18. Douki, T., \& Sage, E. (2016). Dewar valence isomers, the third type of environmentally relevant DNA photoproducts induced by solar radiation. Photochemical \& Photobiological Sciences, 15, 24-30.

19. Mouret, S., Baudouin, C., Charveron, M., Favier, A., Cadet, J., \& Douki, T. (2006). Cyclobutane pyrimidine dimers are predominant DNA lesions in whole human skin exposed to UVA radiation. Proceedings of the National Academy of Sciences of the United States of America, 103, 13765-13770.

20. Bickers, D. R., \& Athar, M. (2006). Oxidative stress in the pathogenesis of skin disease. The Journal of Investigative Dermatology, 126, 2565-2575.

21. Birch-Machin, M. A., \& Bowman, A. (2016). Oxidative stress and ageing. British Journal of Dermatology, 175, S26-29.

22. saes da Silva, E., Tavares, R., da Silva Paulitsch, F., \& Zhang, L. (2018). Use of sunscreen and risk of melanoma and non-melanoma skin cancer: A systemic review and meta-analysis. European Journal of Dermatology, 28, 186-201.

23. Green, A., Williams, G., Neale, R., Hart, V., Leslie, D., Parsons, P., Marks, G. C., Gaffney, P., Battistutta, D., Frost, C., Lang, C., \& Russell, A. (1999). Daily sunscreen application and betacarotene supplementation in prevention of basal-cell and squamous-cell carcinomas of the skin: A randomised controlled trial. Lancet, $354,723-729$

24. Green, A. C., Williams, G. M., Logan, V., \& Strutton, G. M. (2011). Reduced melanoma after regular sunscreen use: Randomized trial follow-up. Journal of Clinical Oncology, 29, 257-263.

25. van der Pols, J. C., Williams, G. M., Pandeya, N., Logan, V., \& Green, A. C. (2006). Prolonged prevention of squamous cell carcinoma of the skin by regular sunscreen use. Cancer Epidemiology, Biomarkers \& Prevention, 15, 2546-2548.

26. Dupont, E., Gomez, J., \& Bilodeau, D. (2013). Beyond UV radiation: A skin under challenge. International Journal of Cosmetic Science, 35, 224-234.

27. Grether-Beck, S., Marini, A., Jaenicke, T., \& Krutmann, J. (2014). Photoprotection in human skin beyond ultraviolet radiation. Photodermatology, Photoimmunology and Photomedicine, 30, 167-174.

28. Zastrow, L., Meinke, M. C., Albrecht, S., Patzeit, A., \& Lademann, J. (2017). From UV protection to protection in the whole spectral range of the solar radiation: New aspects of sunscreen development. Advances in Experimental Medicine and Biology, 996, 311-318.

29. Zastrow, L., Groth, N., Klein, F., Kockott, D., Lademann, J., Renneberg, R., \& Ferrero, L. (2009). The missing link-light-induced
$(280-1.600 \mathrm{~nm})$ free radical formation in human skin. Skin Pharmacology and Physiology, 22, 31-44.

30. Avci, P., Gupta, A., Sadasivam, M., Vecchio, D., Pam, Z., Pam, N. \& Hamblin, M. R. (2013). Low-level laser (light) therapy (LLLT) in skin: stimulating, healing, restoring. In Semin. Cutan. Med. Surg. (Vol. 32, no. 1, pp. 41-52).

31. Narla, S., Kohli, I., Hamzavi, I. H., \& Lim, H. W. (2020). Visible light in photodermatology. Photochemical \& Photobiological Sciences, 19, 99-104.

32. Liebmann, J., Born, M., \& Kolb-Bachofen, V. (2010). Blue-light irradiation regulates proliferation and differentiation in human skin cells. The Journal of Investigative Dermatology, 130, 259-269.

33. Godley, B. F., Shamsi, F. A., Liang, F. Q., Jarrett, S. G., Davies, S., \& Boulton, M. (2005). Blue light induces mitochondrial DNA damage and free radical production in epithelial cells. Journal of Biological Chemistry, 280, 21061-21066.

34. Nakashima, Y., Ohta, S., \& Wolf, A. M. (2017). Blue lightinduced oxidative stress in live skin. Free Radical Biology \& Medicine, 108, 300-310.

35. Mann, T., Eggers, K., Rippke, F., Tesch, M., Buerger, A., Darvin, M. E., Schanzer, S., Meinke, M. C., Lademann, J., \& Kolbe, L. (2020). High-energy visible light at ambient doses and intensities induces oxidative stress of skin-Protective effects of the antioxidant and Nrf2 inducer licochalcone A in vitro and in vivo. Photodermatology, Photoimmunology and Photomedicine, 36, 135-144.

36. Denda, M., \& Fuziwara, S. (2008). Visible radiation affects epidermal permeability barrier recovery: Selective effects of red and blue Light. The Journal of Investigative Dermatology, 128, 1335-1336.

37. Falcone, D., Uzunbajakava, N. E., van Abeelen, F., Oversluizen, G., Peppelman, M., van Erp, P. E. J., \& van de Kerkhof, P. C. M. (2018). Effects of blue light on inflammation and skin barrier recovery following acute perturbation. Pilot study in healthy human subjects. Photodermatology, Photoimmunology \& Photomedicine, 34, 184-193.

38. Regazzetti, C., Sormani, L., Debayle, D., Bernerd, F., Tulic, M. K., De Donatis, G. M., Chignon-Sicard, B., Rocchi, S., \& Passeron, T. (2018). Melanocytes sense blue light and regulate pigmentation through opsin-3. The Journal of Investigative Dermatology, $138,171-178$.

39. Dong, K., Goyarts, E. C., Pelle, E., Trivero, J., \& Pernodet, N. (2019). Blue light disrupts the circadian rhythm and create damage in skin cells. International Journal of Cosmetic Science, 41, $558-562$.

40. Schroeder, P., Calles, C., Benesova, T., Macaluso, F., \& Krutmann, J. (2010). Photoprotection beyond ultraviolet radiationEffective sun protection has to include protection against infrared A radiation-induced skin damage. Skin Pharmacology and Physiology, 23, 15-17.

41. Krutmann, J., Morita, A., \& Chung, J. H. (2012). Sun exposure: What molecular photodermatology tells us about its good and bad sides. The Journal of Investigative Dermatology, 132, 976-984.

42. Akhalaya, M. Y., Maksimov, G. V., Rubin, A. B., Lademann, J., \& Darvin, M. E. (2014). Molecular action mechanisms of solar infrared radiation and heat on human skin. Ageing Research Reviews, $16,1-11$.

43. Kumar, R., Sirohi, T. S., Singh, H., Yadav, R., Roy, R. K., Chaudhary, A., \& Pandeya, S. N. (2014). 1,2,4-triazine analogs as novel class of therapeutic agents. Mini Reviews in Medicinal Chemistry., 14, 168-207.

44. Cascioferro, S., Parrino, B., Spano, V., Carbone, A., Montalbano, A., Barraja, P., Dana, P., \& Cirrincione, G. (2017). An overview on the recent developments of 1,2,4 triazine derivatives as 
anticancer compounds. European Journal of Medicinal Chemistry, 142, 328-375.

45. Verma, T., Sinha, M., \& Bansai, N. (2020). Heterocyclic compounds bearing triazine scaffold and their biological significance: A review. Anti-Cancer Agents in Medicinal Chemistry, 20, 4-28.

46. Diffey, B. L., \& Farr, P. M. (1991). Sunscreen protection against UVB, UVA and blue light: An in vivo and in vitro comparison. British Journal of Dermatology, 124, 258-263.

47. International Organization for Standardization. (2012). ISO 24443: 2012. Determination of sunscreen UVA photoprotection in vitro. International Organization for Standardization.

48. Duteil, L., Cardot-Leccia, N., Queille-Roussel, C., Maubert, Y., Harmelin, Y., Boukari, F., Ambrosetti, D., Lacour, J. P., \& Passeron, T. (2014). Differences in visible light-induced pigmentation according to wavelengths: A clinical and histological study in comparison with UVB exposure. Pigment Cell \& Melanoma Research, 27(5), 822-826.

49. Bacqueville, D., \& Mavon, A. (2008). Caspase-3 activation and DNA damage in pig skin organ culture after solar irradiation. Photochemistry and Photobiology, 84, 1164-1171.

50. Guiraud, B., Hernandez-Pigeon, H., Ceruti, I., Mas, S., Palvadeau, Y., Saint-Martory, C., Castex-Rizzi, N., Duplan, H., \& BessouTouya, S. (2014). Characterisation of a human epidermis model reconstructed from hair follicle keratinocytes and comparison with two commercially models and native skin. International Journal of Cosmetic Science, 36, 485-493.

51. Bacqueville, D., Jacques, C., Duprat, L., Jamin, E. L., Guiraud, B., Perdu, E., Bessou-Touya, S., Zalko, D., \& Duplan, H. (2017). Characterization of xenobiotic metabolizing enzymes of a reconstructed human epidermal model from adult hair follicles. Toxicology and Applied Pharmacology, 329, 190-201.

52. Bacqueville, D., Douki, T., Duprat, L., Rebelo-Moreira, S., Guiraud, B., Dromigny, H., Perier, V., Bessou-Touya, S., \&
Duplan, H. (2015). A new hair follicle-derived human epidermal model for the evaluation of sunscreen genoprotection. Journal of Photochemistry and Photobiology B: Biology, 151, 31-38.

53. Douki, T., von Koschembahr, A., \& Cadet, J. (2017). Insight in DNA repair of UV-induced pyrimidine dimers by chromatographic methods. Photochemistry and Photobiology, 93, 207-215.

54. Terrani, I., Bircher, A. J., \& Scherer Hofmeier, K. (2016). Solar urticaria induced by visible light: Successful treatment with omalizumab. Clinical and Experimental Dermatology, 41, 890-892.

55. Bolognia, J., Jorizzo, J. L., \& Schaffer, J. V. (2012). Dermatology. Elsevier Saunders.

56. Martini, A. P. M., \& Campos, P. M. B. G. M. (2018). Influence of visible light on cutaneous hyperchromias: Clinical efficacy of broad-spectrum sunscreens. Photodermatology, Photoimmunology \& Photomedicine, 34, 241-248.

57. Castanedo-Cazares, J. P., Hernandez-Blanco, D., Carlos-Ortega, B., Fuentes-Ahumada, C., \& Torres-Álvarez, B. (2014). Near visible light and UV photoprotection in the treatment of melasma: A double-blind randomized trial. Photodermatology, Photoimmunology \& Photomedicine, 30, 35-42.

58. Barolet, D., Christiaens, F., \& Hamblin, M. R. (2016). Infrared and skin: Friend or foe. Journal of Photochemistry and Photobiology B: Biology, 155, 78-85.

59. Chamayou-Robert, C., DiGiorgio, C., Brack, O., \& Doucet, O. (2021). Blue light induces DNA damage in normal human skin keratinocytes. Photodermatology, Photoimmunology \& Photomedicine, 00, 1-7.

60. Kielbassa, C., Roza, L., \& Epe, B. (1997). Wavelength dependence of oxidative DNA damage induced by UV and visible light. Carcinogenesis, 18, 811-816.

61. Kielbassa, C., \& Epe, B. (2000). DNA damage induced by ultraviolet and visible light and its wavelength dependence. Methods in Enzymology, 319, 436-445. 